\title{
NUEVA ESCALA DE SEVERIDAD DE LA DMAE. ¿PARA QUÉ SIRVE?
}

\section{NEW ARMD SEVERITY SCALE. WHAT IS IT USED FOR?}

\author{
GARCÍA LAYANA A ${ }^{1}$
}

Todavía tenemos reciente la aparición de una nueva clasificación de la retinopatía diabética, cuando los autores del estudio AREDS (Age-related eye disease study) han propuesto una nueva escala para valorar el riesgo de progresión de los pacientes hacia una DMAE avanzada $(1,2)$. Es importante entender que no se trata solo de clasificar a los pacientes sino más bien de ser capaces de evaluar el riesgo que tienen. Con la irrupción de nuevos tratamientos hemos sido capaces de modificar el curso de esta enfermedad. La terapia fotodinámica, la triamcinolona intravítrea y los nuevos antiangiogénicos de próxima comercialización han permitido que las desastrosas consecuencias de esta enfermedad queden en parte mitigadas. Sin embargo, el reto al que nos enfrentamos en los próximos años es fundamentalmente el de la prevención de la DMAE. Para eso son precisos varios requisitos. En primer lugar un mejor conocimiento de la patogenia del proceso. Los recientes descubrimientos sobre los factores genéticos implicados en esta enfermedad, que apuntan hacia la participación del sistema del complemento, y otros descubrimientos sobre la implicación de diversos factores de crecimiento y del estrés oxidativo son importantes avances en este sentido. En segundo lugar ser capaces de predecir que pacientes con las formas iniciales de la enfermedad son más proclives a acusar una pérdida de visión y por tanto ser objetivo y diana de los tratamientos preventivos. Esto es importante ya que el realizar un tratamiento preventivo en un proceso que en sus formas iniciales afecta a más del $50 \%$ de las personas mayores de 65 años supone un gasto sanitario que convierte esta media en un proceso muy poco efectivo desde el punto de vista económico. En este sentido la nueva clasificación del
AREDS nos puede servir de gran ayuda para acotar mejor que pacientes pueden precisar una terapia preventiva. En tercer lugar, es necesario disponer de tratamientos preventivos eficaces. Hasta la fecha el único tratamiento que ha demostrado eficacia es la suplementación con megadosis de antioxidantes, tal y como demostró el estudio AREDS (3). En este punto hay que recordar que en nuestro país no se dispone de formulaciones comerciales que suministren las dosis que se demostraron efectivas en el estudio antes citado. Se ha iniciado un nuevo estudio AREDS en el que se está evaluando la utilidad de la suplementación con luteina y ácidos grasos omega-3, junto con los anteriores antioxidantes. Será interesante ver los resultados de ese trabajo, el cual no solo nos aclarará el papel que estas dos sustancias tienen en la prevención de la DMAE, sino que además servirá para confirmar o poner en duda los resultados del estudio AREDS-I, el cual no estaba exento de algunas lagunas metodológicas. Se están explorando nuevas estrategias preventivas siendo una de las más interesantes, el empleo de Acetato de Anecortave por vía subtenoniana cada seis meses en el ojo contralateral de pacientes que ya han desarrollado una DMAE avanzada en uno de sus ojos (4). El efecto de esta sustancia sobre las metaloproteinasas de matriz, uno de los elementos que interviene de manera más precoz en la formación de la neovascularización coroidea, hace que su empleo sea lógico al menos desde un punto de vista teórico.

En cuanto a la nueva clasificación, hay que señalar que en realidad se han diseñado dos clasificaciones $(1,2)$. Una más compleja, que está diseñada fundamentalmente para el seguimiento de pacientes dentro de ensayos clínicos (1). Existe otra más simplificada, que nos puede ayudar, si no a contestar al

\footnotetext{
1 Doctor en Medicina. Pamplona.

E-mail: aglayana@unav.es
} 
menos a informar mejor, cuando nos hacen la enojosa pregunta de «si pasará lo mismo en el otro ojo».

Esta clasificación simplificada ha determinado 5 niveles que cuantifican el riesgo de progresión en cinco años de ojos con formas iniciales de DMAE a formas avanzadas.

La escala suma elementos de riesgo presentes en la retina de ambos ojos. Se suma «un punto» por la presencia de una o más drusas de tamaño grande (de al menos 125 micras, o lo que es lo mismo, equivalentes al diámetro de una vena retiniana a la altura del margen de la papila). Si este tipo de drusas está presente en los dos ojos la puntuación será de «dos puntos». Si no hay drusas grandes en ninguno de los ojos pero hay drusas intermedias (de al menos 65 micras) se da «un punto». Igualmente se da «un punto» por la presencia de alteraciones del epitelio pigmentado cuando están presentes en un ojo y «dos puntos» si son bilaterales. La DMAE avanzada en un ojo se puntúa como «dos puntos»y los factores anteriores solo se evalúan en el ojo contralateral. En total podremos sumar entre 0 y 4 puntos. Esto equivale a un riesgo que se cuantifica de la siguiente manera: 0 puntos equivalen a un riesgo en cinco años de $0,5 \%$. Un punto equivale a $3 \% ; 2$ puntos equivalen al 12\%; 3 puntos equivalen al $25 \%$ y 4 puntos equivalen al $50 \%$.

En la escala simplificada el valor que tiene la existencia de una DMAE avanzada en un ojo queda minusvalorado respecto a la importancia que se le da en la escala más compleja de nueve niveles (1). Otro inconveniente que tiene esta escala es que emplea el tamaño de drusa por razones prácticas en vez del área total de drusas que es un valor que tie- ne una mayor capacidad predictiva de riesgo (1). Un tercer inconveniente es que no se tiene en cuenta otros factores de riesgo sistémicos como hipertensión arterial tabaco, cirugía de catarata, predisposición genética, o índice de masa corporal (5).

Por tanto, aunque esta escala puede y probablemente será mejorada en el futuro, a la luz de los nuevos descubrimientos, puede ser una herramienta muy útil a la hora de valorar a aquellos pacientes cuyo riesgo de progresión de su DMAE es más elevado.

\section{BIBLIOGRAFÍA}

1. Davis MD, Gangnon RE, Lee LY, Hubbard LD, Klein BE, Klein R, et al. The Age-Related Eye Disease Study severity scale for age-related macular degeneration: AREDS Report No. 17. Arch Ophthalmol 2005; 123: 1484-1498.

2. Ferris FL, Davis MD, Clemons TE, Lee LY, Chew EY, Lindblad AS, et al. A simplified severity scale for age-related macular degeneration: AREDS Report No. 18. Arch Ophthalmol 2005; 123: 1570-1574.

3. Age-Related Eye Disease Study Research Group. A randomized, placebo-controlled, clinical trial of high-dose supplementation with vitamins $C$ and $E$, beta carotene, and zinc for age-related macular degeneration and vision loss: AREDS report no. 8. Arch Ophthalmol 2001; 119: 1417-1436.

4. Russell SR, Slakter JS, Ho AC, Kaiser PK, Regillo CD, Schmidt-Erfurth UM, et al. Anecortave Acetate Treatment of "Dry" AMD to Reduce Risk of Progression to "Wet" AMD-The Anecortave Acetate Risk Reduction Trial (AART). Invest Ophthalmol Vis Sci 2004; 45: Abstract 3134.

5. Clemons TE, Milton RC, Klein R, Seddon JM, Ferris FL 3rd; Age-Related Eye Disease Study Research Group. Risk factors for the incidence of Advanced Age-Related Macular Degeneration in the Age-Related Eye Disease Study (AREDS) AREDS report no. 19. Ophthalmology 2005; 112: 533-539. 